\title{
Optimal Deployment of Autonomous Vehicle Lanes with Endogenous Market Penetration
}

\author{
Zhibin Chen ${ }^{\mathrm{a}}$, Fang $\mathrm{He}^{\mathrm{b}}$, Lihui Zhang ${ }^{\mathrm{c}, *}$ and Yafeng Yin ${ }^{\mathrm{a}}$ \\ ${ }^{a}$ Department of Civil and Coastal Engineering, University of Florida, 365 Weil Hall, Gainesville, \\ FL 32611-6580, United States \\ ${ }^{b}$ Department of Industrial Engineering, Tsinghua University, N502 Shunde Building, \\ Beijing, 100084, P.R. China \\ ${ }^{c}$ College of Civil Engineering and Architecture, Zhejiang University, B815 Anzhong Building, \\ Hangzhou, 310058, P.R. China
}

\begin{abstract}
This paper develops a mathematical approach to optimize a time-dependent deployment plan of autonomous vehicle (AV) lanes on a transportation network with heterogeneous traffic stream consisting of both conventional vehicles (CVs) and AVs, so as to minimize the social cost and promote the adoption of AVs. Specifically, AV lanes are exclusive lanes that can only be utilized by AVs, and the deployment plan specifies when, where, and how many AV lanes to be deployed. We first present a multi-class network equilibrium model to describe the flow distributions of both CVs and AVs, given the presence of AV lanes in the network. Considering that the net benefit (e.g., reduced travel cost) derived from the deployment of AV lanes will further promote the $\mathrm{AV}$ adoption, we proceed to apply a diffusion model to forecast the evolution of AV market penetration. With the equilibrium model and diffusion model, a timedependent deployment model is then formulated, which can be solved by an efficient solution algorithm. Lastly, numerical examples based on the south Florida network are presented to demonstrate the proposed models.

Keywords: autonomous vehicle; autonomous-vehicle lane; market penetration; deployment plan
\end{abstract}

* Corresponding author. Tel.: 86-571-88208968; Email: lihuizhang@zju.edu.cn 


\section{Introduction}

Due to potential benefits on traffic safety, driver productivity, road capacity, travel speed, energy consumption, and vehicular emission (Shladover et al., 2012; Greenblatt and Saxena, 2015; Levin and Boyles, 2016a,b; Mersky and Samaras, 2016), autonomous vehicles (AVs) have attracted tremendous attentions. Recent progress suggests AVs are on the horizon. Since 2009 when Google started testing self-driving technology in California, Google's AVs have already achieved a total mileage over 1.5 million miles (Google Self-Driving Car Project, 2016). The National Highway Traffic Safety Administration (NHTSA) of the United States has agreed to consider the Google self-driving computer system as the "driver" of the vehicle (NHTSA, 2016). Besides Google, many car manufactures, such as Volvo, BMW and Audi, are testing their prototype AVs. More recently, Japanese government announced that AVs could be used to ferry people around Tokyo during the 2020 Olympics and Paralympics (2025AD, 2016).

Despite all these exciting developments, it will still be many years for AVs to be widely adopted, and the heterogeneous traffic stream consisting of both conventional vehicles (CVs) and AVs will inevitably exist for a long time. To promote the adoption of AVs, efforts on both technical level and policy level are of critical importance. The former mainly refers to the development of AV technology primarily driven by private sectors (e.g., Google), and the latter refers to policies proposed by government agencies to adapt to the deployment of AV technology. From the policy aspect, apart from legalizing on-road AV test driving, the government agencies may need to identify proper locations to implement AV mobility applications, and enhance dedicated lanes, segments and areas for AVs. For example, some regular lanes can be converted into dedicated AV lanes, which can only be used by AVs. As demonstrated by Tientrakool et al. (2011), the capacity of those lanes will approximately become tripled due to the benefits (e.g., reduced inter-vehicle safe distance) resulted from vehicle-to-vehicle communication.

Accordingly, deploying AV lanes can be expected to help AVs save trip times, which can further boost the market penetration of AVs and reduce the system delay. On the other hand, conversion of regular lanes to AV lanes may result in increased trip times of CVs due to their loss of accessibility to those AV lanes, and thus may damage the social welfare.

This paper attempts to propose a general mathematical model to help government agencies optimally deploy AV lanes in a way to minimize the social cost. The decision-making process in such a planning practice possesses a structure of the leader-follower or Stackelberg game, in which government agencies serve as the leader and travelers are the follower. In order for 
government agencies to optimize those planning decisions, travelers' spontaneous responses need to be proactively considered in the optimization framework. This type of Stackelberg games have been formulated as mathematical programs with equilibrium constraints for many transportation applications (see, e.g., Wu et al., 2011, 2012; Yin et al., 2008; He et al., 2013, 2015; Zhang et al., 2014; Chen et al., 2016). More specifically, given AV lanes deployed, we assume that CVs and AVs follow the Wardrop equilibrium principle to choose their routes that minimize their individual travel costs (Wardrop, 1952), and the resulting flow distribution is in a multi-class network equilibrium (e.g., Yang and Meng, 2001; Wu et al., 2006). Furthermore, since the net benefit (e.g., reduced travel cost for AVs) derived from deploying AV lanes plays an important role in promoting the AV adoption, we apply a diffusion model to forecast the evolution of AV market penetration. Based on the network equilibrium model and diffusion model, we proposed a time-dependent deployment model to optimize the location design of AV lanes on a general transportation network. The AV market penetration follows a progressive process instead of a radical one, thus the AV lanes should also be deployed in a progressive fashion. More specifically, the optimized deployment plan will not only specify where and how many AV lanes to be deployed, but also when to deploy them.

For the remainder, Section 2 applies the multi-class network equilibrium model to describe the flow distributions of both CVs and AVs. Section 3 proposes the AV diffusion model to forecast the market penetration of AVs. Section 4 presents the mathematical program to optimize the AV-lane deployment plan, followed by numerical examples in Section 5. Concluding remarks are provided in the last section.

Below are some notations used throughout the paper. 


\begin{tabular}{|c|c|}
\hline \multicolumn{2}{|l|}{ Sets } \\
\hline$K$ & Set of paired links \\
\hline$N$ & Set of nodes \\
\hline$A$ & Set of links \\
\hline$\hat{A}$ & Set of AV links \\
\hline$M$ & Set of travel modes: mode 1 denotes CVs, and mode 2 denotes AVs \\
\hline$W$ & Set of origin-destination (OD) pairs \\
\hline$P_{\tau}^{w, m}$ & Set of paths for travel mode $m \in M$ between OD pair $w \in W$ at year $\tau \in T$ \\
\hline$\hat{P}_{\tau}^{w, m}$ & $\begin{array}{l}\text { Set of utilized paths for travel mode } m \in M \text { between OD pair } w \in W \text { at year } \\
\tau \in T\end{array}$ \\
\hline$T$ & Set of years \\
\hline \multicolumn{2}{|c|}{ Parameters } \\
\hline$m$ & Index of travel mode, $m \in M$ \\
\hline$w$ & Index of OD pair, $w \in W$ \\
\hline$p$ & Index of path, $p \in P_{\tau}^{w, m}$ \\
\hline$d^{w *}$ & Potential AV market size for OD pair $w \in W$ \\
\hline$\gamma_{m}$ & Value of time (VOT) for drivers of travel mode $m \in M$ \\
\hline$\sigma$ & Interest rate \\
\hline$n$ & A factor converting social cost from an hourly basis to a yearly basis \\
\hline$\tau$ & Index of year $\tau \in T$ \\
\hline$\varsigma$ & Unsafety factor for using CV \\
\hline$\theta_{a}^{k}$ & $\begin{array}{l}\text { If link } a \text { belongs to the } k \text { th link pair, and it is an } \mathrm{AV} \text { link, then } \theta_{a}^{k}=1 \text {; If link } a \\
\text { belongs to the } k \text { th link pair, and it is not an } \mathrm{AV} \text { link, then } \theta_{a}^{k}=-1 \text {; otherwise, } \\
\theta_{a}^{k}=0\end{array}$ \\
\hline \multicolumn{2}{|c|}{ Variables } \\
\hline$d_{\tau}^{w, m}$ & Demand of travel mode $m \in M$ between OD pair $w \in W$ at year $\tau \in T$ \\
\hline$x_{a, \tau}^{w, m}$ & $\begin{array}{l}\text { Flow of travel mode } m \in M \text { on link } a \in A \text { between OD pair } w \in W \text { at year } \\
\tau \in T\end{array}$ \\
\hline$v_{a, \tau}$ & Aggregate flow on link $a \in A$ at year $\tau \in T$ \\
\hline$y_{\tau}^{k}$ & $\begin{array}{l}\text { The number of lanes on the } k \text { th link pair that are converted into AV lanes at } \\
\text { year } \tau \in T\end{array}$ \\
\hline$C_{\tau}^{w, m}$ & $\begin{array}{l}\text { Equilibrium travel time for mode } m \in M \text { between OD pair } w \in W \text { at year } \\
\tau \in T\end{array}$ \\
\hline
\end{tabular}

\section{Multi-Class Network Equilibrium Model}

Assume that the entire planning horizon is divided into $|T|$ years. Let $G(N, A)$ denote a general transportation network, where $N$ and $A$ are the sets of nodes and links in the network respectively. Let $\hat{A}$ represent the set of AV links in the network. Note that any link including AV lanes can be divided into one regular link and one AV link without affecting the network performance. For example, Figure 1(a) shows a simple network topology. If we consider link 1 and link 4 as the candidate links where AV lanes can be deployed, then its network topology can be revised as the 
one in Figure 1(b). That is, $A=\{1,2,3,4,5,6,7\}$ and $\hat{A}=\{6,7\}$. We further define $K$ as the set of these pairs of links. Specifically, in Figure 1(b), $K=\{(1,6),(4,7)\}$. We represent a link either as $a \in A$ or its starting and ending nodes, i.e., $a=(i, j) \in A$. Let $M=\{1,2\}$ denote the set of travel modes, in which mode 1 corresponds to $\mathrm{CV}$ and mode 2 corresponds to AV. The set of OD pairs is denoted as $W$, and $o(w)$ and $d(w)$ define the origin and destination of OD pair $w \in W$. The travel time of link $a \in A$ at year $\tau \in \Gamma$ is denoted as $t_{a, \tau}\left(v_{a, \tau}\right)$, which is specified by the link performance function, e.g., in a form of the following function:

$$
t_{a, \tau}\left(v_{a, \tau}\right)=t_{a}^{0}\left[1+\bar{\alpha}_{a}\left(\frac{v_{a, \tau}}{\Lambda_{a}^{\tau}}\right)^{\bar{\beta}_{a}}\right]
$$

where $t_{a}^{0}$ is the free-flow travel time of link $a ; \Lambda_{a}^{\tau}$ is the capacity of link $a$ at year $\tau \in T ; v_{a, \tau}$ is the link flow at year $\tau \in T$, and $\bar{\alpha}_{a}$ and $\bar{\beta}_{a}$ are two positive parameters.



(a) Original network topology

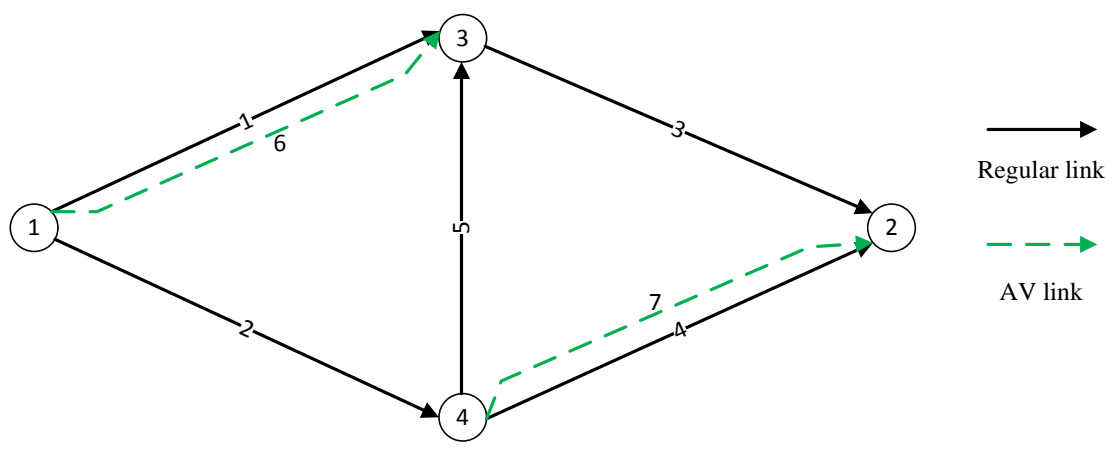

(b) Revised network topology

Figure 1 A simple network example

The flow distributions of both CVs and AVs at any year $\tau \in T$, can be described by the following network equilibrium model: 


$$
\begin{array}{cl}
\Delta \mathbf{x}_{\tau}^{w, m}=\mathbf{E}^{w, m} d_{\tau}^{w, m} & \forall w \in W, m \in M \\
x_{a, \tau}^{w, 2} \geq 0 & \forall a \in A, w \in W \\
x_{a, \tau}^{w, 1} \geq 0 & \forall a \in A \backslash \hat{A}, w \in W \\
x_{a, \tau}^{w, 1}=0 & \forall a \in \hat{A}, w \in W \\
v_{a, \tau}=\sum_{m \in M} \sum_{w \in W} x_{a, \tau}^{w, m} & \forall a \in A \\
t_{a, \tau}\left(v_{a, \tau}\right)+\rho_{i, \tau}^{w, m}-\rho_{j, \tau}^{w, m}-\eta_{a, \tau}^{w, m}=0 & \forall a \in A, w \in W, m \in M \\
\eta_{a, \tau}^{w, 2} \cdot x_{a, \tau}^{w, 2}=0 & \forall a \in A, w \in W \\
\eta_{a, \tau}^{w, 1} \cdot x_{a, \tau}^{w, 1}=0 & \forall a \in A \backslash \hat{A}, w \in W \\
\eta_{a, \tau}^{w, 2} \geq 0 & \forall a \in A, w \in W \\
\eta_{a, \tau}^{w, 1} \geq 0 & \forall a \in A \backslash \hat{A}, w \in W
\end{array}
$$

where $\Delta$ is the node-link incidence matrix associated with a given network, and $\mathbf{E}^{w, m}, w \in$ $W, m \in M$ is a vector with a length of $|N|$. The vector consists of two non-zero components: one has a value of 1 corresponding to origin $o(w)$ and the other has a value of -1 corresponding to destination $s(w) . x_{a, \tau}^{w, m}$ is the link flow of mode $m \in M$ between O-D pair $w \in W$ at year $\tau \in T$, and $v_{a, \tau}$ is the aggregation of $x_{a, \tau}^{w, m}$ over all travel modes and OD pairs. Vectors $\boldsymbol{\rho}$ and $\boldsymbol{\eta}$ are auxiliary variables, and $\boldsymbol{\rho}$ represents the node potentials.

In the above, constraint (1) ensures the flow conservation; constraints (2) and (3) are nonnegative constraints on link flows; constraint (4) ensures that only AVs can use AV links; constraint (5) aggregates link flows across all travel modes and OD pairs; constraints (6)-(10) ensure that all utilized paths of the same travel mode between each OD pair share the same travel $\operatorname{cost} \rho_{s(w), \tau}^{w, m}-\rho_{o(w), \tau}^{w, m}$, while those unutilized ones possess travel cost larger than or equal to $\rho_{s(w), \tau}^{w, m}-\rho_{o(w), \tau}^{w, m}$.

In addition, finding a solution to the system of equilibrium conditions is equivalent to solving the following mathematical problem (NE):

NE:

$$
\min _{\mathbf{x}} \sum_{a \in A} \int_{0}^{v_{a, \tau}} t_{a, \tau}(x) d x
$$

s.t. (1)-(5) 
The equivalence can be established by comparing the KKT conditions of NE with the defined network equilibrium conditions (1)-(10).

\section{AV Diffusion Model}

Diffusion models have been widely applied to forecast how a new product or idea will be adopted over time. For example, Yang and Meng (2001) proposed a modified logistic growth model to investigate the adoption rate of advanced traveler information systems. Park et al. (2011) proposed a diffusion model to simulate the market penetration of hydrogen fuel cell vehicles. Lavasani et al. (2016) developed a market penetration model to forecast the AV technology adoption by considering the price difference between AV and CV, as well as the economic wealth of the population. We here adopt the diffusion model proposed by Yang and Meng (2001). Specifically, the adoption of AVs at a given year depends on the adoption and the net benefit gained at the previous year. That is,

$$
d_{\tau+1}^{w, 2}=d_{\tau}^{w, 2}+\mathrm{g}\left(\phi_{\tau}^{w}\right) d_{\tau}^{w, 2}\left(1-\frac{d_{\tau}^{w, 2}}{d^{w *}}\right) \quad \forall w \in W, \tau \in T \backslash\{|T|\}
$$

where $d^{w *}$ is the potential AV market size for OD pair $w \in W$. Note that, the potential market size of a new product is predicted exogenously in many diffusion models (e.g., Lavasani et al., 2016; Park et al, 2011; Massiani and Gohs, 2015), with a few exception (Yang and Meng, 2001; Huang and $\mathrm{Li}, 2007)$. The latter ones relate the potential market penetration level to the benefit brought by the new product. Doing so, however, will complicate the AV-lane deployment model (proposed in Section 4), and even make it intractable. Therefore, in this paper, we adopt a fixed potential AV market size for each OD pair. $\mathrm{g}\left(\phi_{\tau}^{w}\right)$ is the intrinsic variable growth coefficient for OD pair $w \in W$, which is defined as follows:

$$
\mathrm{g}\left(\phi_{\tau}^{w}\right)=\hat{a} e^{\hat{b}\left(\phi_{\tau}^{w}-\bar{\phi}^{w}\right)} \quad \forall w \in W, \tau \in T
$$

where $\hat{a}$ and $\hat{b}$ are two parameters $(\hat{a}>0 ; \hat{b} \geq 0), \bar{\phi}^{w}$ is the OD specific benefit threshold for OD pair $w \in W$, and $\phi_{\tau}^{w}$ is the net benefit gained for OD pair $w \in W$ at year $\tau \in T$. $\phi_{\tau}^{w}$ is defined as follows:

$$
\phi_{\tau}^{w}=\left[\left(\gamma_{1}+\varsigma\right) C_{\tau}^{w, 1}-\gamma_{2} C_{\tau}^{w, 2}\right] \cdot L_{\tau}^{w}-Y_{\tau} \quad \forall w \in W, \tau \in T
$$

where $\gamma_{m}$ is the value of travel time for travel mode $m, \varsigma$ is a nonnegative unsafety factor for using CVs compared with using $\mathrm{AVs}, L_{\tau}^{w}$ is the number of trips between OD pair $w \in W$ at year $\tau \in T$, which could be the average annual trip number obtained from household travel survey, $Y_{\tau}$ 
is the additional annual cost for using AVs at year $\tau \in T$, and $C_{\tau}^{w, m}$ is the equilibrium travel time of mode $m \in M$ between OD pair $w \in W$ at year $\tau \in T$, i.e.,

$$
C_{\tau}^{w, m}=\rho_{s(w), \tau}^{w, m}-\rho_{o(w), \tau}^{w, m} \quad \forall m \in M, w \in W, \tau \in T
$$

where $\boldsymbol{\rho}$ can be obtained by solving NE.

Without loss of generality, we assume that the yearly travel demand between each OD pair remains the same during the entire planning horizon. That is,

$$
\sum_{m \in M} d_{\tau}^{w, m}=\sum_{m \in M} d_{0}^{w, m} \quad \forall w \in W, \tau \in T
$$

\section{AV-Lane Location Problem}

In this section, we will investigate how to optimally locate AV lanes to minimize the social cost with the consideration of the market penetration of AVs. AV lanes can only be located to a given set of candidate links, to reflect possible restrictions imposed in field applications. The optimal deployment problem of AV lanes will be formulated as a bi-level model. The lower-level problem is the multi-class network equilibrium defined in Eqs. (1)-(10), while the upper-level one investigates when, where and how many AV lanes should be deployed.

\subsection{Model formulation}

Let $\theta_{a}^{k}$ denote the pair-link incidence. If link $a$ belongs to the $k$ th pair of links, and it is an AV link, then $\theta_{a}^{k}=1$; if it is a regular link, then $\theta_{a}^{k}=-1$; otherwise, $\theta_{a}^{k}=0$. Further, let $y_{\tau}^{k}$ be an integer variable, representing the number of lanes on the $k$ th pair of links that are converted from regular lanes to AV lanes at year $\tau$. Then, the AV-lane location problem (AVLL) can be formulated as follows:

AVLL:

$$
\min _{\mathbf{x}, \mathbf{d}, \boldsymbol{\eta}, \boldsymbol{\rho}, \mathbf{y}} \sum_{\tau \in T} \sum_{w \in W}\left[\sum_{m \in M} n \frac{\left(\left(\gamma_{1}+\varsigma\right) d_{\tau}^{w, 1} C_{\tau}^{w, 1}+\gamma_{2} d_{\tau}^{w, 2} C_{\tau}^{w, 2}\right)}{(1+\sigma)^{\tau-1}}\right]
$$

s.t. (1)-(15) 


$$
\begin{array}{cl}
\Lambda_{a}^{\tau}=\bar{\Lambda}_{a}+\bar{c}_{a} \cdot \sum_{k \in K} \theta_{a, k} \sum_{j=1}^{\tau} y_{j}^{k} & \forall a \in A, \tau \in T \\
\bar{\Lambda}_{a}+\bar{c}_{a} \cdot \sum_{k \in K} \theta_{a, k} \sum_{j=1}^{|T|} y_{j}^{k} \geq \mu_{a} & \forall a \in A \\
y_{\tau}^{k} \in\left\{0,1, \ldots, I_{k}\right\} & \forall k \in K, \tau \in T
\end{array}
$$

where $\sigma$ is the discount rate per year, $n$ is a factor converting social cost from an hourly basis to a yearly basis, $\mu_{a}$ is a given parameter, representing the minimum capacity required for link $a, I_{k}$ is a given integer, representing the maximum number of $\mathrm{AV}$ lanes that can be deployed on the $k$ th pair of links each year, $\bar{\Lambda}_{a}$ is the initial capacity of link $a, \bar{c}_{a}$ is the per-lane capacity of link $a$, thus $\bar{\Lambda}_{a}+\bar{c}_{a} \cdot \sum_{k \in K} \theta_{a, k} \sum_{j=1}^{\tau} y_{k, j}$ represents the capacity of link $a$ at year $\tau$. It should be noted that the increase of AV-link capacity and the decrease of the paired regular-link capacity is not symmetric, as their per-lane capacities are not the same. As mentioned before, the per-lane capacity can become tripled when it is converted from a regular lane to an AV lane due to the benefits from vehicle-to-vehicle communication.

In the above, the objective function is to minimize the total social cost, consisting of the costs of both CVs and AVs; constraint (16) calculates the capacity of link $a$ at year $\tau$; constraint (17) ensures that the capacity of link $a$ should be no less than a required minimum capacity. For example, in order to maintain the accessibility of the network, there must be at least one regular lane for all the regular links, otherwise, CVs of some OD pairs cannot finish their trips. Constraint (18) implies that $y_{\tau}^{k}$ must be an integer number, and its upper bound is $I_{k}$.

The above model can be readily extended to consider the construction cost for the AV-lane deployment and the government subsidy, via adding a term $\sum_{\tau} \frac{\Pi_{\tau}}{(1+\sigma)^{\tau-1}}-\sum_{\tau} \frac{S_{\tau}}{(1+\sigma)^{\tau-1}}$ to the objective function, where $\Pi_{\tau}$ and $S_{\tau}$ are the construction cost and the government subsidy at year $\tau$ respectively.

\subsection{Solution algorithm}

The AVLL problem can be generally categorized as a discrete network design problem (DNDP). And those solution algorithms proposed in the literature for DNDP can be employed to solve AVLL, e.g., branch-and-bound technique (LeBlanc, 1975), support-function based method (Gao et al., 2005), active-set algorithm (Zhang et al., 2009), system optimal-relaxation based method 
and user equilibrium-reduction based method (Wang et al., 2013). Here, AVLL is in form of a mathematical program with complementarity constraints (see, e.g., Luo et al., 1996), we employ the active-set algorithm developed by Zhang et al. (2009) to solve it. The basic idea is to solve a sequence of restricted nonlinear problems to obtain a strongly stationary solution to the original AVLL.

Let $\Phi_{k}$ denote the smallest integer number such that $I_{k} \leq 2^{\Phi_{k}}-1$, then constraint (18) can be represented as $y_{\tau}^{k}=\sum_{\varpi=1}^{\Phi_{k}} y_{\tau}^{k, \varpi} 2^{\varpi-1}$, where $y_{\tau}^{k, \varpi}$ is a binary variable for $\varpi \in\left\{1, \ldots, \Phi_{k}\right\}$.

For a particular deployment plan, we define $|T|$ pairs of active sets, $\Omega_{\tau, 0}=\left\{(k, \varpi): y_{\tau}^{k, \varpi}=\right.$ $0\}$ and $\Omega_{\tau, 1}=\left\{(k, \varpi): y_{\tau}^{k, \varpi}=1\right\}, \forall \tau \in T$. These two sets should be "complete", i.e., $\Omega_{\tau, 0} \cup$ $\Omega_{\tau, 1}=\{(k, \varpi)\} ; \Omega_{\tau, 0} \cap \Omega_{\tau, 1}=\emptyset, \forall \tau \in T$. Given some deployment plan $\cup_{\tau \in \Gamma}\left(\Omega_{\tau, 0}, \Omega_{\tau, 1}\right)$, the restricted AVLL (RAVLL) problem can be formulated as below:

RAVLL:

$$
\min _{\boldsymbol{x}, \boldsymbol{d}, \boldsymbol{\eta}, \boldsymbol{\rho}, \boldsymbol{y}} \sum_{\tau \in T} \sum_{w \in W}\left[\sum_{m \in M} n \frac{\left(\left(\gamma_{1}+\varsigma\right) d_{\tau}^{w, 1} C_{\tau}^{w, 1}+\gamma_{2} d_{\tau}^{w, 2} C_{\tau}^{w, 2}\right)}{(1+\sigma)^{\tau-1}}\right]
$$

s.t. (1)-(15)

$$
\begin{array}{cl}
\Lambda_{a}^{\tau}=\bar{\Lambda}_{a}+\bar{c}_{a} \cdot \sum_{k \in K} \theta_{a, k} \sum_{j=1}^{\tau} \sum_{\varpi}^{\Phi_{k}} y_{j}^{k, \varpi} & \forall a \in A, \tau \in T \\
\bar{\Lambda}_{a}+\bar{c}_{a} \cdot \sum_{k \in K} \theta_{a, k} \sum_{j=1}^{|T|} \sum_{\varpi}^{\Phi_{k}} y_{j}^{k, \varpi} \geq \mu_{a} & \forall a \in A \\
y_{\tau}^{k, \varpi}=0 & \forall(k, \varpi) \in \Omega_{\tau, 0}, \tau \in T \\
y_{\tau}^{k, \varpi}=1 & \forall(k, \varpi) \in \Omega_{\tau, 1}, \tau \in T
\end{array}
$$

Although RAVLL is another mathematical problem with complementarity constraints, its optimal solution can be easily obtained by solving the NE problem, with the deployment plan fixed. Below is the procedure of the active-set algorithm. The convergence of the algorithm has been proved by Zhang et al. (2009), thus is not presented here.

Step 0: Set $\epsilon=1$ and solve NE with an initial deployment plan $\cup_{\tau \in T}\left(\Omega_{\tau, 0}^{1}, \Omega_{\tau, 1}^{1}\right)$ for each year $\tau \in T$. 
Step 1: Construct a solution $(\mathbf{x}, \mathbf{d}, \boldsymbol{\eta}, \boldsymbol{\rho}, \mathbf{y})^{T}$ to RAVLL based on the optimal solutions derived from solving NE with $\cup_{\tau \in T}\left(\Omega_{\tau, 0}^{\epsilon}, \Omega_{\tau, 1}^{\epsilon}\right)$. Then, solve RAVLL to determine $\lambda_{k, \varpi, \tau}^{\epsilon}$ and $\mu_{k, \varpi, \tau}^{\epsilon}$, the Lagrangian multipliers associated with constraints (21) and (22). Set $T T^{\epsilon}=$ $\sum_{\tau \in T} \sum_{w \in W}\left[\sum_{m \in M} n \frac{\left(\left(\gamma_{1}+\varsigma\right) d_{\tau}^{w, 1} c_{\tau}^{w, 1}+\gamma_{2} d_{\tau}^{w, 2} c_{\tau}^{w, 2}\right)}{(1+\sigma)^{\tau-1}}\right]$.

Step 2: Set $Q=-\infty$ and adjust the active sets by performing the following steps:

a) Let $(\hat{\mathbf{z}}, \hat{\mathbf{h}})$ solve the following knapsack problem:

$$
\min \sum_{\tau \in T} \sum_{(k, \varpi) \in \Omega_{\tau, 0}^{\epsilon}} \lambda_{k, \varpi, \tau}^{\epsilon} z_{k, \varpi, \tau}-\sum_{\tau \in T} \sum_{(k, \varpi) \in \Omega_{\tau, 1}^{\epsilon}} \mu_{k, \varpi, \tau}^{\epsilon} h_{k, \varpi, \tau}
$$

s.t.

$$
\begin{aligned}
& \bar{\Lambda}_{a}+\bar{c}_{a} \cdot \sum_{\tau \in T}[\left.\sum_{(k, \varpi) \in \Omega_{\tau, 1}^{\epsilon}} \theta_{a, k} 2^{\varpi-1}+\sum_{(k, \varpi) \in \Omega_{\tau, 0}^{\epsilon}} \theta_{a, k} z_{k, \varpi, \tau} 2^{\varpi-1}-\sum_{(k, \varpi) \in \Omega_{\tau, 1}^{\epsilon}} \theta_{a, k} h_{k, \varpi, \tau} 2^{\varpi-1}\right] \\
& \geq \mu_{a}, \forall a \in A \\
& \sum_{\tau \in T} \sum_{(k, \varpi) \in \Omega_{\tau, 0}^{\epsilon}} \lambda_{k, \varpi, \tau}^{\epsilon} z_{k, \varpi, \tau}-\sum_{\tau \in T} \sum_{(k, \varpi) \in \Omega_{\tau, 1}^{\epsilon}} \mu_{k, \varpi, \tau}^{\epsilon} h_{k, \varpi, \tau} \geq Q \\
& z_{k, \varpi, \tau}, h_{k, \varpi, \tau} \in\{0,1\}
\end{aligned}
$$

If its optimal objective value is zero, stop and the current solution is optimal.

Otherwise, go to Step 2b.

b) Set:

i. $\quad D=\sum_{\tau \in T} \sum_{(k, \varpi) \in \Omega_{\tau, 0}^{\epsilon}} \lambda_{k, \varpi, \tau}^{\epsilon} \hat{z}_{k, \varpi, \tau}-\sum_{\tau \in \Gamma} \sum_{(k, \varpi) \in \Omega_{\tau, 1}^{\epsilon}} \mu_{k, \varpi, \tau}^{\epsilon} \hat{h}_{k, \varpi, \tau}$,

ii. $\quad \widehat{\Omega}_{\tau, 0}=\left(\Omega_{\tau, 0}^{\epsilon}-\left\{(k, \varpi) \in \Omega_{\tau, 0}^{\epsilon}: \hat{z}_{k, \varpi, \tau}=1\right\}\right) \cup\left\{(k, \varpi) \in \Omega_{\tau, 1}^{\epsilon}: \hat{h}_{k, \varpi, \tau}=\right.$ $1, \forall \tau \in T$,

iii. $\quad \widehat{\Omega}_{\tau, 1}=\left(\Omega_{\tau, 1}^{\epsilon}-\left\{(k, \varpi) \in \Omega_{\tau, 1}^{\epsilon}: \hat{h}_{k, \varpi, \tau}=1\right\}\right) \cup\left\{(k, \varpi) \in \Omega_{\tau, 0}^{\epsilon}: \hat{z}_{k, \varpi, \tau}=\right.$ 1, $\forall \tau \in T$.

c) Solve NE with a deployment plan $\hat{\mathbf{y}}$ compatible with $\cup_{\tau \in T}\left(\widehat{\Omega}_{\tau, 0}, \widehat{\Omega}_{\tau, 1}\right)$. If its social cost $T T<T T^{\epsilon}$, go to Step $2 \mathrm{~d}$ since the location plan $\cup_{\tau \in T}\left(\widehat{\Omega}_{\tau, 0}, \widehat{\Omega}_{\tau, 1}\right)$ leads to a decrease in the social cost. Otherwise, set $Q=D+\varepsilon$, where $\varepsilon>0$ is sufficiently small, and return to Step 2a. 
d) Set $\Omega_{\tau, 0}^{\epsilon+1}=\widehat{\Omega}_{\tau, 0}, \Omega_{\tau, 1}^{\epsilon+1}=\widehat{\Omega}_{\tau, 1}, \forall \tau \in T$, and $\epsilon=\epsilon+1$. Go to Step 1 .

\section{Numerical Examples}

\subsection{Basic settings}

The numerical examples are conducted based on the south Florida network as shown in Figure 2, which consists of 232 regular links, 44 AV links, 82 nodes and 83 OD pairs. The OD demand is given in Table 1 and link characteristics are omitted due to space limitation. Table 2 shows the paired links, in which each AV link is paired with one regular link. For example, link 233 is an AV link, and link 15 is the paired regular link. They have the same link characteristics except the initial number of lanes and per-lane capacity. Specifically, the initial capacities of AV links are set as 0 , meaning that without deploying AV lanes, the AV links are only virtual links, which can not be utilized.

We assume that the initial adoption rate of AVs for each OD pair is $2 \%$, and the potential market size is $75 \%$ (Lavasani et al., 2016). The default model parameters include: (1) discount rate: $\sigma=0.03$; (2) converting factor: $n=365 \times 24=8,760$ (hour/year); (3) per-lane capacity of a regular link: $\bar{c}_{a}, \forall a \in A \backslash \hat{A}$, equal to the link capacity divided by the number of lanes on that link; (4) per-lane capacity of an AV link: $\bar{c}_{a}, \forall a \in \hat{A}$, equal to 2.5 times the per-lane capacity of the paired regular link; (5) planning horizon: $|T|=40$; (6) the number of trips: $L_{\tau}^{W}=720$ (trips/year), $\forall w \in W, \tau \in \Gamma$; (7) additional annual cost for using AVs: $Y_{\tau}=1,000$ (\$/year), $\forall \tau \in \Gamma$; (8) OD specific benefit threshold: $\bar{\phi}^{w}=1,000$ (\$), $\forall w \in W$; (9) VOT: $\gamma_{1}=0.5$ and $\gamma_{2}=0.5(\$ / \mathrm{min}) ;(10)$ unsafety factor for using CV: $\varsigma=0.1(\$ / \mathrm{min}) ;(11)$ two parameters in Eq. (11): $\hat{a}=0.3$ (1/year), $\hat{b}=0.00005$ (year/\$); (12) minimum link capacity: $\mu_{a}=\bar{c}_{a}, \forall a \in$ $A \backslash \hat{A}$, and $\mu_{a}=0, \forall a \in \hat{A}$; (13) maximum number of AV lanes can be deployed each year: $I_{k}=3, \forall k \in K$. It should be noted that all the above values are chosen for illustrative purpose. 


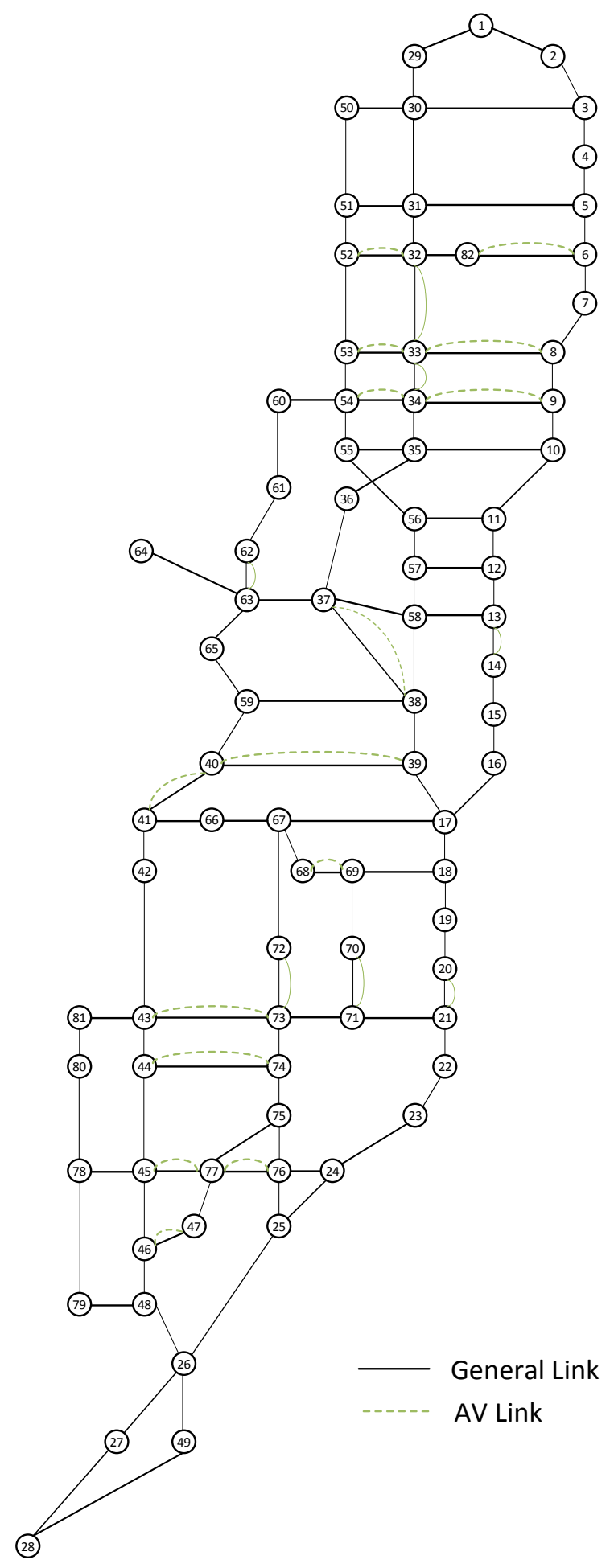

Figure 2 South Florida network 
Table 1 OD demand of south Florida network

\begin{tabular}{llllllll}
\hline OD & Demand & OD & Demand & OD & Demand & OD & Demand \\
\hline $1-36$ & 743.56 & $28-57$ & 743.56 & $50-19$ & 793.76 & $64-30$ & 815.30 \\
$1-57$ & 860.80 & $28-63$ & 863.41 & $50-59$ & 758.15 & $66-31$ & 768.05 \\
$4-64$ & 810.61 & $29-37$ & 794.11 & $50-69$ & 806.96 & $68-5$ & 801.23 \\
$5-40$ & 837.18 & $29-62$ & 806.96 & $51-21$ & 804.53 & $70-82$ & 802.10 \\
$5-41$ & 862.89 & $31-70$ & 770.49 & $51-23$ & 760.76 & $74-8$ & 826.94 \\
$6-42$ & 823.64 & $32-24$ & 763.02 & $52-44$ & 768.92 & $74-33$ & 843.44 \\
$7-72$ & 809.91 & $32-76$ & 848.65 & $52-71$ & 757.29 & $75-33$ & 832.32 \\
$8-47$ & 847.60 & $32-80$ & 824.16 & $53-24$ & 820.68 & $76-8$ & 777.95 \\
$9-46$ & 847.08 & $33-74$ & 752.60 & $53-46$ & 798.97 & $76-33$ & 842.74 \\
$10-45$ & 825.72 & $34-48$ & 812.35 & $53-75$ & 766.84 & $76-53$ & 816.17 \\
$12-28$ & 810.09 & $36-1$ & 845.87 & $54-45$ & 835.45 & $78-35$ & 828.85 \\
$13-2$ & 823.98 & $40-30$ & 789.77 & $54-78$ & 841.53 & $78-53$ & 769.79 \\
$14-1$ & 854.38 & $41-51$ & 846.91 & $55-48$ & 765.62 & $78-55$ & 759.89 \\
$19-4$ & 843.26 & $43-7$ & 802.79 & $55-79$ & 862.37 & $81-8$ & 767.19 \\
$19-50$ & 856.46 & $44-82$ & 864.97 & $57-1$ & 832.84 & $81-33$ & 845.00 \\
$21-51$ & 861.33 & $45-54$ & 803.49 & $58-29$ & 774.83 & $81-52$ & 826.07 \\
$24-53$ & 786.64 & $46-53$ & 745.82 & $60-1$ & 836.84 & $82-22$ & 763.89 \\
$24-82$ & 797.93 & $48-8$ & 812.00 & $61-1$ & 746.69 & $82-42$ & 838.40 \\
$26-9$ & 825.72 & $48-55$ & 768.75 & $61-27$ & 782.30 & $82-74$ & 811.30 \\
$26-10$ & 781.78 & $49-10$ & 749.82 & $61-49$ & 815.12 & $82-80$ & 766.67 \\
$28-56$ & 839.27 & $49-34$ & 865.49 & $63-29$ & 776.22 & & \\
\hline
\end{tabular}

Table 2 AV links and their paired links

\begin{tabular}{ccccccccc}
\hline Pair & $\begin{array}{c}\text { AV } \\
\text { link }\end{array}$ & $\begin{array}{c}\text { Paired } \\
\text { link }\end{array}$ & Pair & $\begin{array}{c}\text { AV } \\
\text { link }\end{array}$ & $\begin{array}{c}\text { Paired } \\
\text { link }\end{array}$ & Pair & $\begin{array}{c}\text { AV } \\
\text { link }\end{array}$ & $\begin{array}{c}\text { Paired } \\
\text { link }\end{array}$ \\
\hline 1 & 233 & 15 & 16 & 248 & 94 & 31 & 263 & 178 \\
2 & 234 & 20 & 17 & 249 & 102 & 32 & 264 & 180 \\
3 & 235 & 23 & 18 & 250 & 105 & 33 & 265 & 194 \\
4 & 236 & 34 & 19 & 251 & 111 & 34 & 266 & 196 \\
5 & 237 & 36 & 20 & 252 & 112 & 35 & 267 & 199 \\
6 & 238 & 52 & 21 & 253 & 113 & 36 & 268 & 201 \\
7 & 239 & 53 & 22 & 254 & 116 & 37 & 269 & 204 \\
8 & 240 & 84 & 23 & 255 & 123 & 38 & 270 & 205 \\
9 & 241 & 85 & 24 & 256 & 127 & 39 & 271 & 207 \\
10 & 242 & 87 & 25 & 257 & 130 & 40 & 272 & 209 \\
11 & 243 & 88 & 26 & 258 & 133 & 41 & 273 & 217 \\
12 & 244 & 89 & 27 & 259 & 135 & 42 & 274 & 218 \\
13 & 245 & 90 & 28 & 260 & 147 & 43 & 275 & 221 \\
14 & 246 & 91 & 29 & 261 & 150 & 44 & 276 & 231 \\
15 & 247 & 92 & 30 & 262 & 153 & & & \\
\hline
\end{tabular}




\subsection{Plan comparison}

In this section, we consider three different deployment plans to demonstrate how an appropriate plan can benefit the system performance. The first plan is to do nothing, meaning that no AV lanes will be deployed; the second plan is listed in Table 3; and the third plan is to deploy all the AV lanes in Table 3 at the first year (see Table 4). The social costs associated with these three plans are calculated to be $\$ 6.845 \times 10^{11}, \$ 6.582 \times 10^{11}$, and $\$ 6.814 \times 10^{11}$, respectively. As can be observed, although the number of AV lanes and their locations are exactly the same for plan 2 and plan 3, the performance of plan 2 is much better than that of plan 3 in term of the social cost. Compared with plan 1 (to do nothing), the former reduces the social cost by $3.84 \%$, while the latter only leads to a reduction of $0.45 \%$. It implies that considering the time dimension into the deployment plan is of critical importance.

Table 3 Deployment plan 2

\begin{tabular}{ccccccccc}
\hline Pair & $\tau$ & $\begin{array}{c}\text { Number of AV } \\
\text { lanes deployed }\end{array}$ & Pair & $\tau$ & $\begin{array}{c}\text { Number of AV } \\
\text { lanes deployed }\end{array}$ & Pair & $\tau$ & $\begin{array}{c}\text { Number of AV } \\
\text { lanes deployed }\end{array}$ \\
\hline 2 & 21 & 1 & 13 & 10 & 1 & 22 & 11 & 1 \\
3 & 10 & 1 & 13 & 11 & 1 & 22 & 12 & 1 \\
4 & 11 & 1 & 14 & 12 & 1 & 23 & 25 & 2 \\
4 & 12 & 3 & 15 & 9 & 1 & 26 & 22 & 1 \\
5 & 11 & 3 & 15 & 11 & 1 & 27 & 35 & 1 \\
5 & 12 & 1 & 16 & 13 & 1 & 29 & 13 & 2 \\
5 & 17 & 1 & 17 & 10 & 1 & 30 & 13 & 1 \\
8 & 10 & 1 & 17 & 11 & 1 & 37 & 35 & 3 \\
8 & 11 & 1 & 18 & 10 & 1 & 37 & 36 & 1 \\
10 & 35 & 1 & 18 & 11 & 1 & 37 & 37 & 1 \\
10 & 36 & 1 & 19 & 10 & 1 & 39 & 35 & 3 \\
11 & 10 & 1 & 20 & 15 & 1 & 39 & 36 & 2 \\
11 & 11 & 1 & 21 & 12 & 1 & 42 & 1 & 2 \\
12 & 11 & 2 & 21 & 13 & 1 & & & \\
\hline
\end{tabular}


Table 4 Deployment plan 3

\begin{tabular}{ccccccccc}
\hline Pair & $\tau$ & $\begin{array}{c}\text { Number of AV } \\
\text { lanes deployed }\end{array}$ & Pair & $\tau$ & $\begin{array}{c}\text { Number of AV } \\
\text { lanes deployed }\end{array}$ & Pair & $\tau$ & $\begin{array}{c}\text { Number of AV } \\
\text { lanes deployed }\end{array}$ \\
\hline 2 & 1 & 1 & 14 & 1 & 1 & 23 & 1 & 2 \\
3 & 1 & 1 & 15 & 1 & 2 & 26 & 1 & 1 \\
4 & 1 & 4 & 16 & 1 & 1 & 27 & 1 & 1 \\
5 & 1 & 5 & 17 & 1 & 2 & 29 & 1 & 2 \\
8 & 1 & 2 & 18 & 1 & 2 & 30 & 1 & 1 \\
10 & 1 & 2 & 19 & 1 & 1 & 37 & 1 & 5 \\
11 & 1 & 2 & 20 & 1 & 1 & 39 & 1 & 5 \\
12 & 1 & 2 & 21 & 1 & 2 & 42 & 1 & 2 \\
13 & 1 & 2 & 22 & 1 & 2 & & & \\
\hline
\end{tabular}

We further examine the evolution of AV market penetration and the annual cost under the three plans, as displayed in Figure 3 and Figure 4. It can be observed that the adoption rate resulted from plan 3 grows the fastest, which is easy to understand since plan 3 provides all the capacity for AVs at the very beginning of the modeling horizon. The annual costs for the first four years under plan 3 are much higher than those under the other two plans. The reason behind is when the level of market penetration of AVs is low, although deploying all the AV lanes can help to enlarge the gain of this portion of vehicles, it will lead to tremendous increase in the travel time of CVs. As a result, the total social welfare decreases. What's worse, as shown in Figure 4, such negative effect can last for several years as it takes time for CV drivers to adopt AVs. On the contrary, although plan 2 does not promote the adoption rate as quickly as plan 3, it does reduce the social cost by a larger amount via deploying AV lanes progressively. It is worthwhile to highlight that, in plan 2, most of the AV lanes are deployed after the $10^{\text {th }}$ year when the AV market penetration is high enough, i.e., 26\% (see Figure 3). When the market penetration of $\mathrm{AVs}$ is low (e.g., at the first several years), only two AV lanes are deployed (see Table 3). 


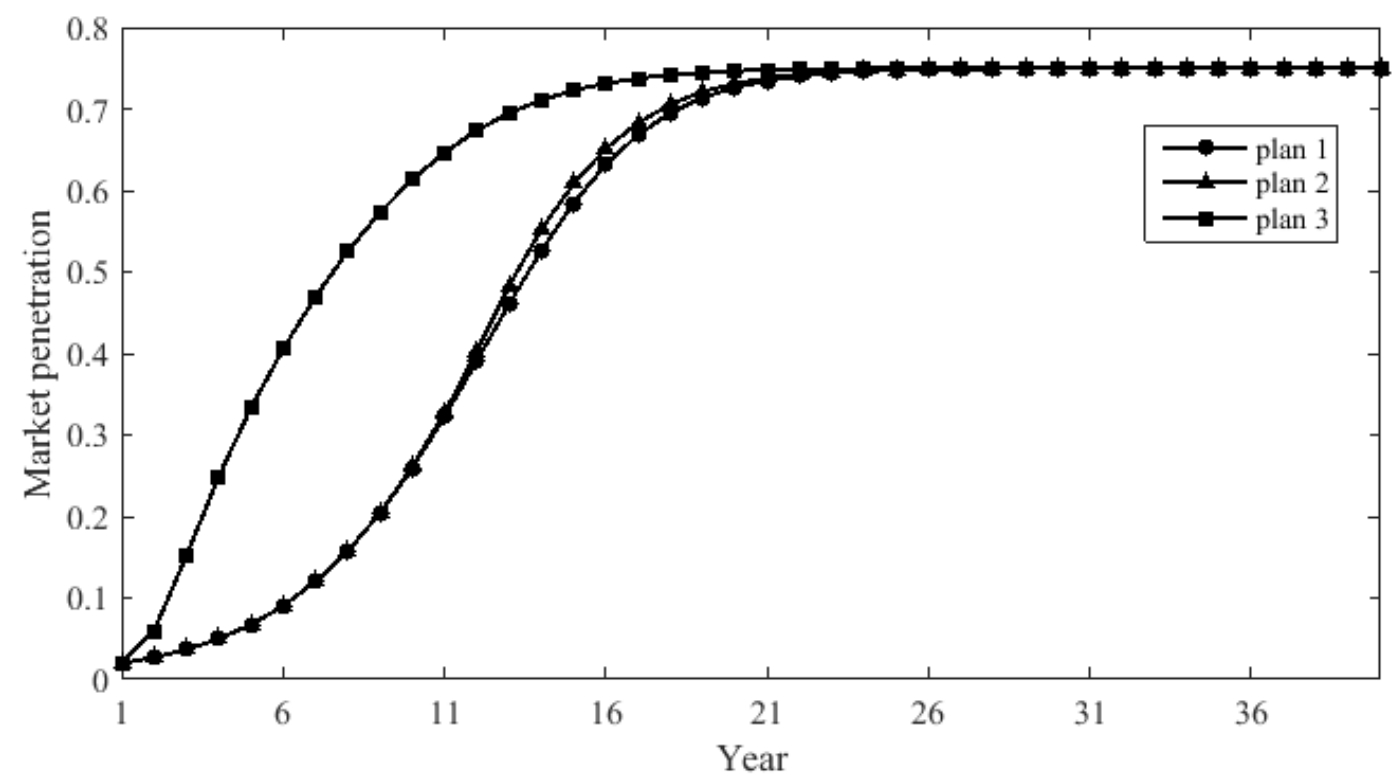

Figure 3 Evolution of AV market penetration under various plans

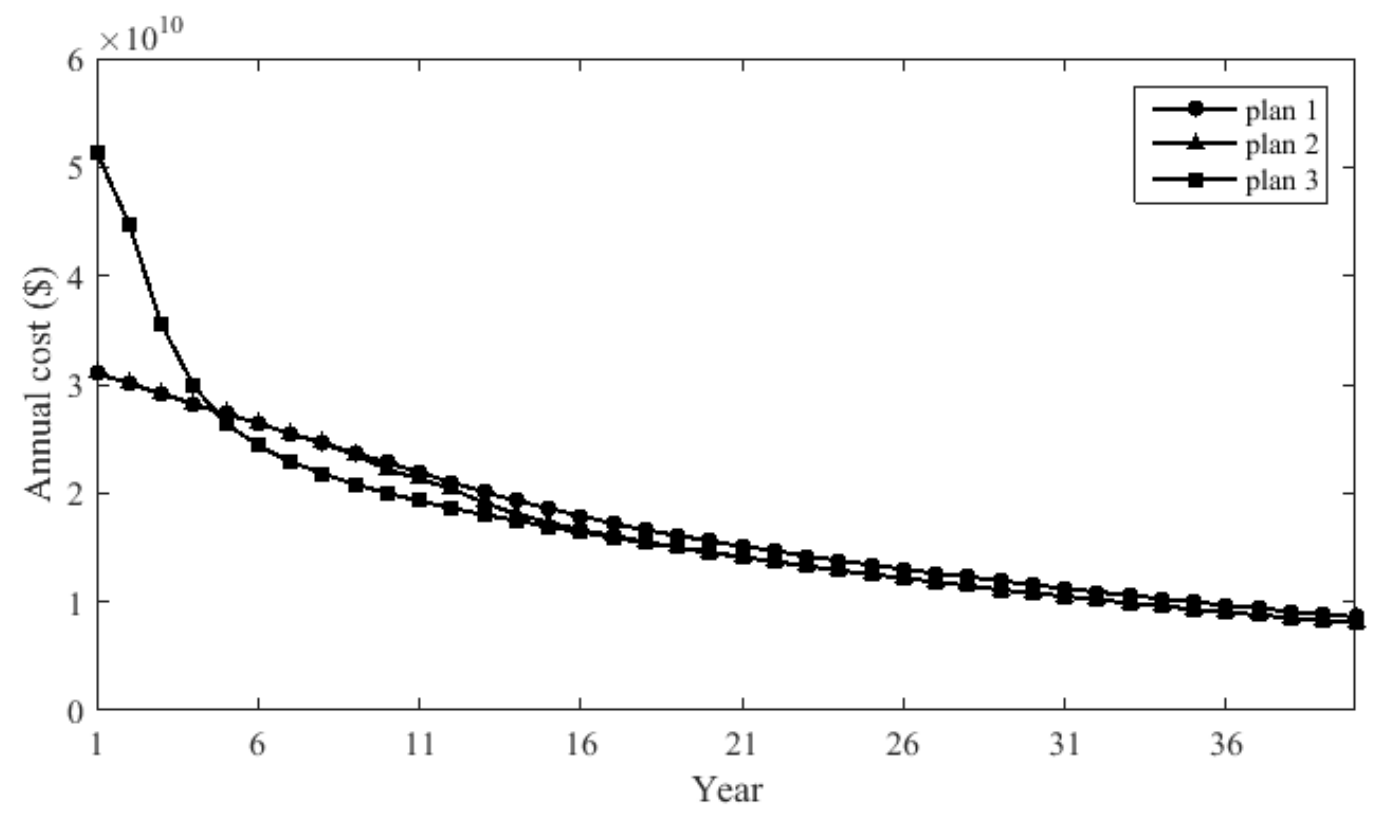

Figure 4 Evolution of annual cost under various plans

\subsection{Sensitivity analyses}

As many parameters have impact on the market penetration of AVs, sensitivity analyses are conducted in this section. All the numerical experiments in this section are based on plan 2 . 
Figure 5 shows the $\mathrm{AV}$ market penetration curves with variable capacity ratios between $\mathrm{AV}$ lanes and regular lanes. Specifically, "3.0 times" means that the per-lane capacity becomes tripled when it is converted from a regular lane to an AV lane. Interestingly, although the growth rate increases as the capacity ratio increases, the differences among them are indistinctive in Figure 5, which indicates that increasing the per-lane capacity of AV lanes will not significantly promote the market penetration. It makes sense because the coverage area of AV links is relatively small, thus increasing their capacities only leads to limited reduction in the AVs' trip times. Actually, the total social costs associated with " 1.5 times", " 2.0 times", "2.5 times", and “3.0 times" are $\$ 6.693 \times 10^{11}, \$ 6.619 \times 10^{11}, \$ 6.582 \times 10^{11}$, and $\$ 6.562 \times 10^{11}$, respectively. The variance is very small.

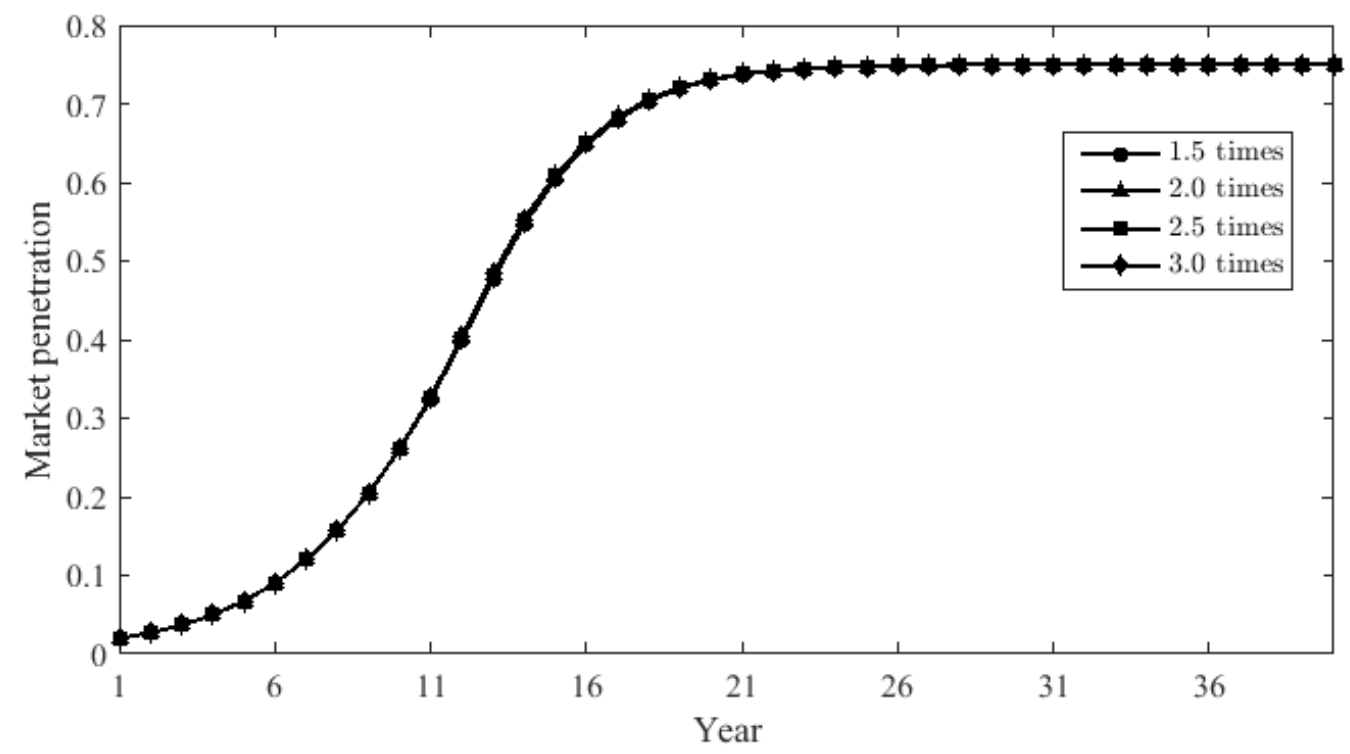

Figure 5 Evolution of AV market penetration with variable ratios of AV-lane capacity over regularlane capacity

Figure 6 specifies the evolution of AV market penetration with different unsafety factors (i.e., $\varsigma)$. As $\varsigma$ increases, the growth rate increases, and it takes fewer years to reach the potential market size. The reason is straightforward: when the unsafety factor of using CVs becomes larger, the incentive for people to adopt AVs will be higher. 


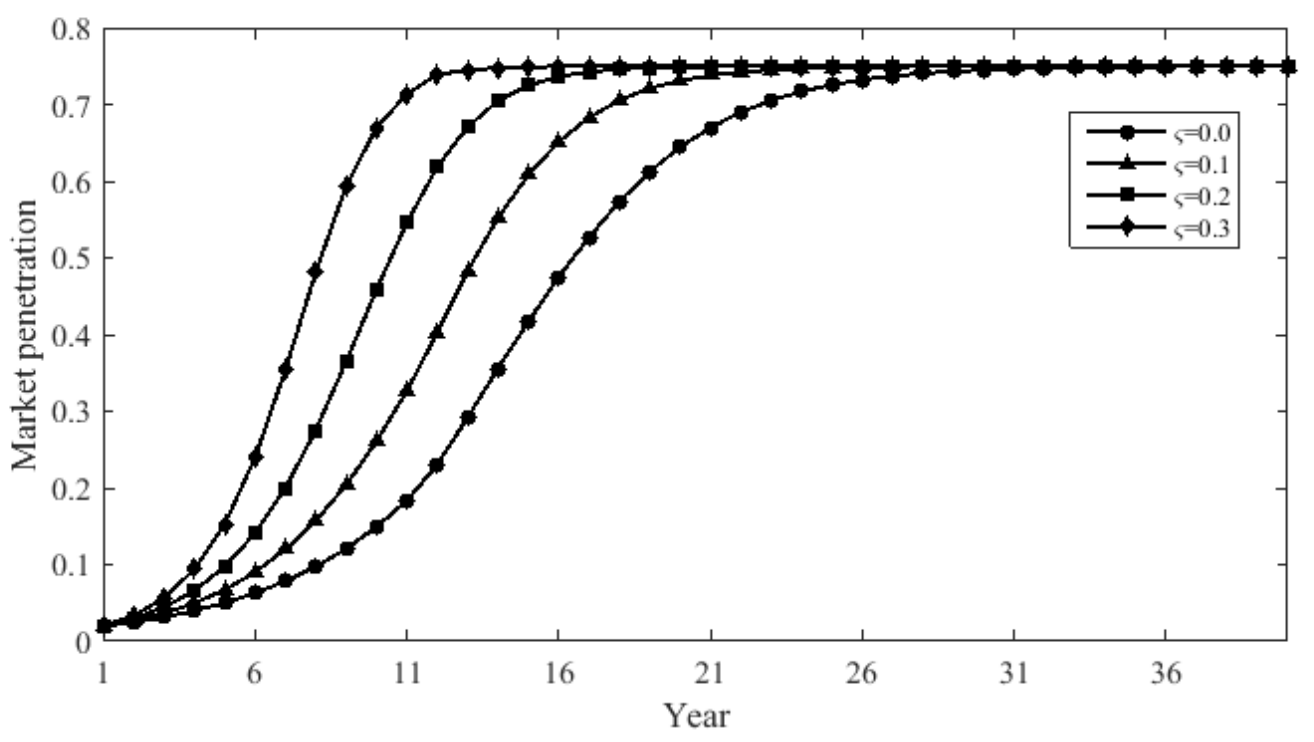

Figure 6 Evolution of AV market penetration with different unsafety factors

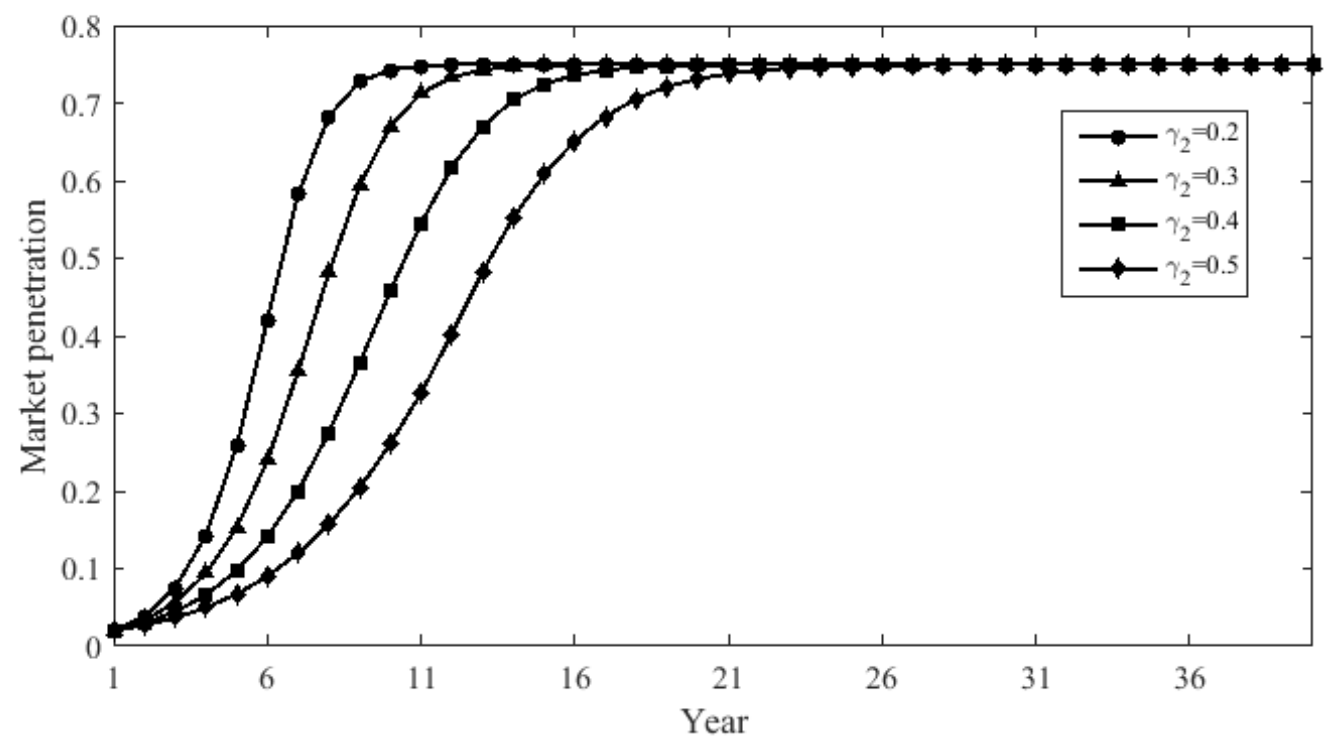

Figure 7 Evolution of AV market penetration with different VOTs of AVs

Traveling with AVs, people can concentrate on dealing with other personal matters instead of driving, thus their VOTs (i.e., $\gamma_{2}$ ) are envisioned to be no greater than those traveling with CVs (i.e., $\gamma_{1}$ ). To examine how $\gamma_{2}$ affects the AV adoption rate, Figure 7 plots the penetration curves with various $\gamma_{2}$. It can be observed that as $\gamma_{2}$ increases, the growth rate increases, and the time to reach the saturation point becomes shorter. For example, when $\gamma_{2}=0.2(\$ / \mathrm{min})$, it only takes 12 
years to reach the saturation point, which is only half of the time needed when $\gamma_{2}=0.5(\$ / \mathrm{min})$. Accordingly, we may expect that the higher autonomous level of AVs is, the higher adoption rate will be.

To enable full-autonomous driving, intelligent control systems and various types of sensors (e.g., cameras, radar, and ultrasonic sensors) are required. Consequently, AVs are usually more expensive to use than $\mathrm{CVs}$, and the additional costs become a critical factor preventing people from adopting AVs. Figure 8 describes how the evolution curve of AV market penetration changes with changing additional annual cost. As expected, higher additional annual costs will lead to lower growth rates. However, the saturation points do not vary much with different additional annual costs. Specifically, it takes about 26 years to achieve the potential market size for all scenarios.

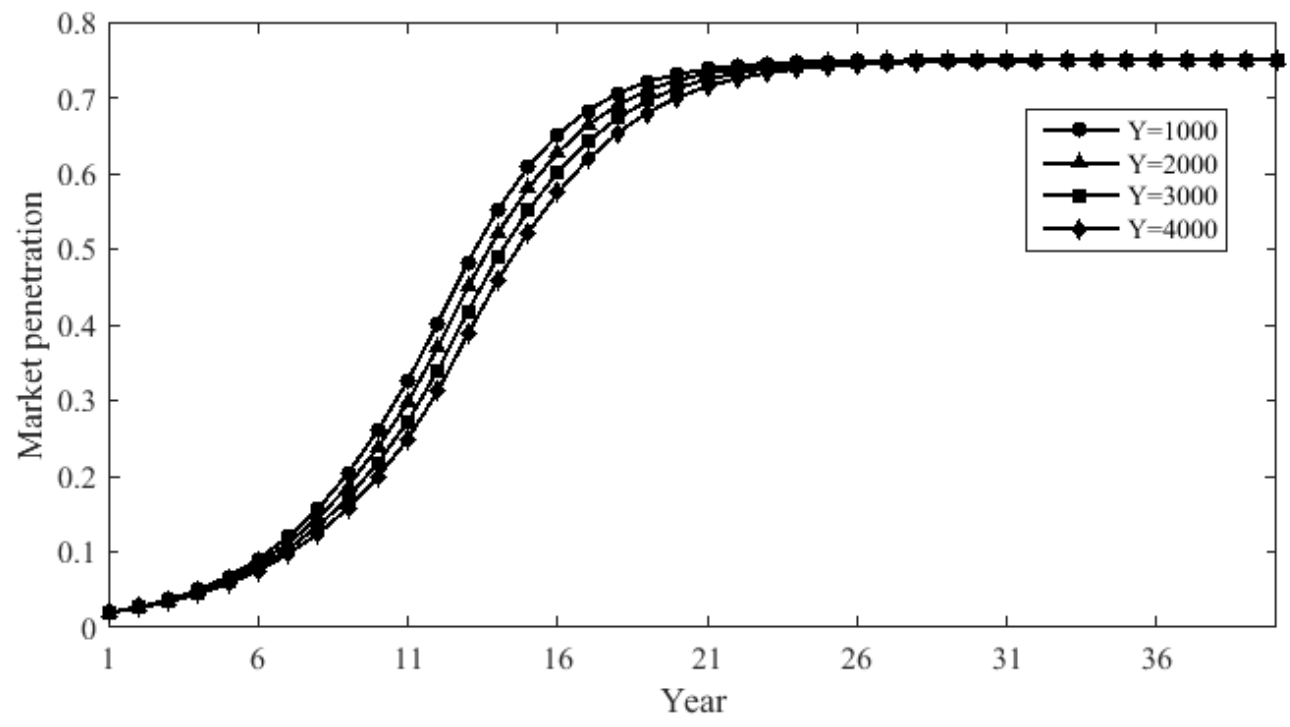

Figure 8 Evolution of AV market penetration with different additional annual costs for using AVs

As the number of annual trips varies from person to person, Figure 9 illustrates its impact on the AV market evolution. As can be seen, increasing the number of annual trips results in increased adoption rate of AVs, as well as fewer years to reach the saturation point. This is because more benefit can be derived when more trips are involved as per Eq. (13).

To investigate the impact of the potential market size on the market penetration curve, simulation experiments based on four potential market sizes: $65 \%, 75 \%, 85 \%$, and $95 \%$ are conducted. Figure 10 illustrates the associated evolution patterns of AV market penetration. All 
patterns have similar growth rates in the earlier years (e.g., year 1 to10), while the growth rates diverge in the later years, and higher potential market sizes lead to larger growth rates. It is worthwhile to point out that the saturation points associated with different potential market sizes do not vary much, which is in agreement with the finding of Lavasani et al. (2016)



Figure 9 Evolution of AV market penetration with different numbers of annual trips

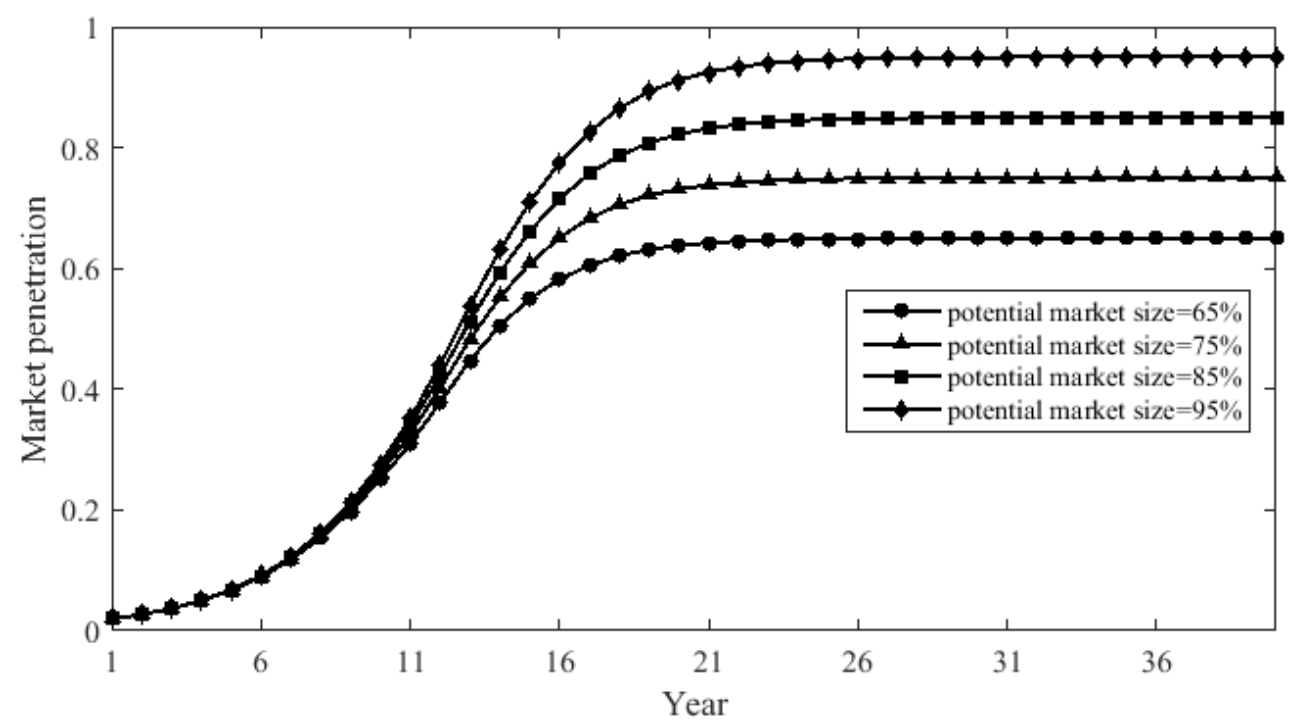

Figure 10 Evolution of AV market penetration with different potential market sizes 


\subsection{Optimal location plan}

In this section, we solve AVLL for the south Florida network. Instead of starting with only one initial deployment plan, we start with different initial plans for the active-set algorithm, and take the best optimal plan as the final solution. By doing so, some poor local solution can be avoided. The final deployment plan obtained is given in Table 5, and the associated social cost is $\$ 6.578 \times 10^{11}$. Compared with plan 1 (to do nothing), the optimal plan reduces the social cost by $\$ 2.674 \times 10^{10}$ or $3.91 \%$. Figure 11 and Figure 12 illustrate the evolution of AV market penetration and annual cost under both the optimal plan and plan 1. As we can see, the optimal plan does not lead to reduced annual cost until the $9^{\text {th }}$ year, when the AV market penetration reaches a relative high rate.

Table 5 Optimal deployment plan

\begin{tabular}{ccccccccc}
\hline Pair & $\tau$ & $\begin{array}{c}\text { Number of AV } \\
\text { lanes deployed }\end{array}$ & Pair & $\tau$ & $\begin{array}{c}\text { Number of AV } \\
\text { lanes deployed }\end{array}$ & Pair & $\tau$ & $\begin{array}{c}\text { Number of AV } \\
\text { lanes deployed }\end{array}$ \\
\hline 2 & 21 & 2 & 13 & 10 & 2 & 21 & 12 & 2 \\
3 & 1 & 1 & 14 & 12 & 1 & 22 & 9 & 1 \\
4 & 12 & 3 & 15 & 8 & 1 & 22 & 11 & 1 \\
4 & 26 & 1 & 15 & 11 & 1 & 23 & 25 & 2 \\
5 & 11 & 3 & 16 & 13 & 1 & 26 & 22 & 1 \\
5 & 13 & 2 & 17 & 10 & 2 & 29 & 13 & 2 \\
8 & 9 & 2 & 18 & 10 & 2 & 30 & 13 & 1 \\
11 & 10 & 2 & 19 & 10 & 1 & 42 & 1 & 1 \\
12 & 11 & 2 & 20 & 15 & 1 & 42 & 2 & 1 \\
\hline
\end{tabular}




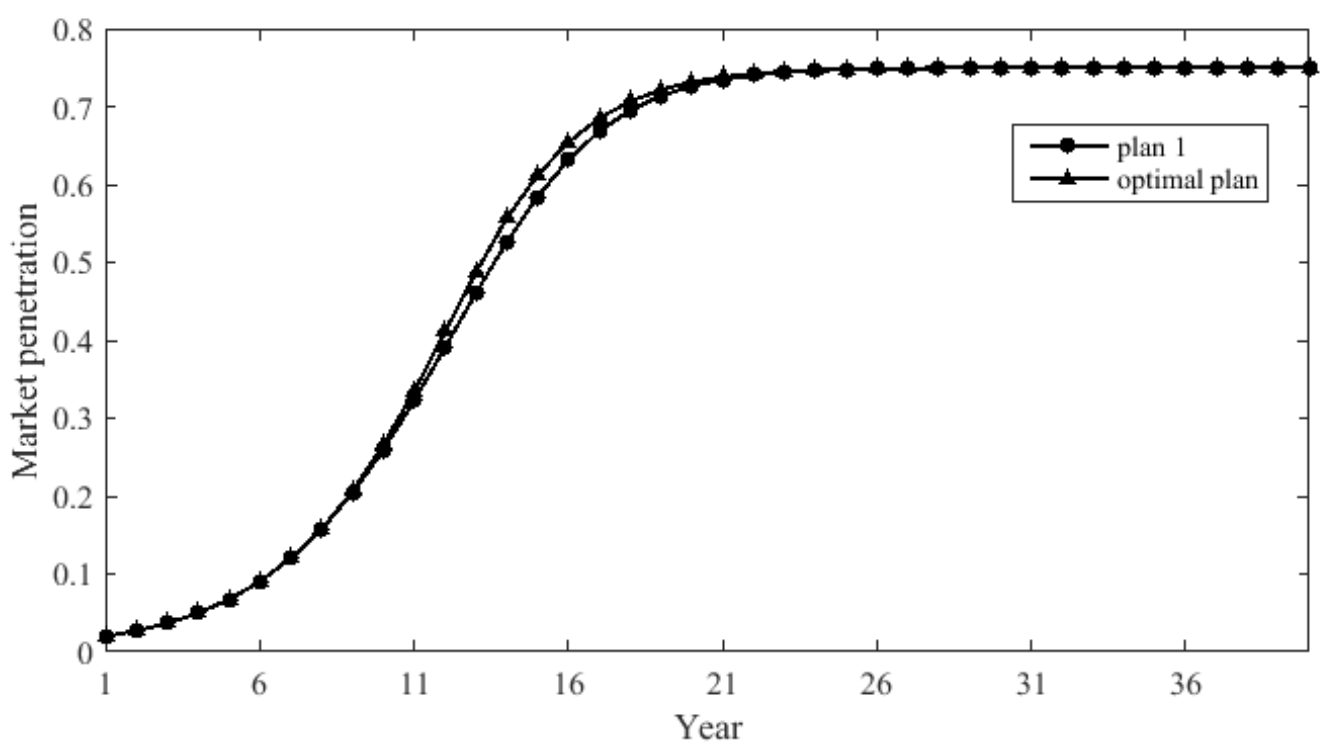

Figure 11 Evolution of AV market penetration under plan 1 and the optimal plan

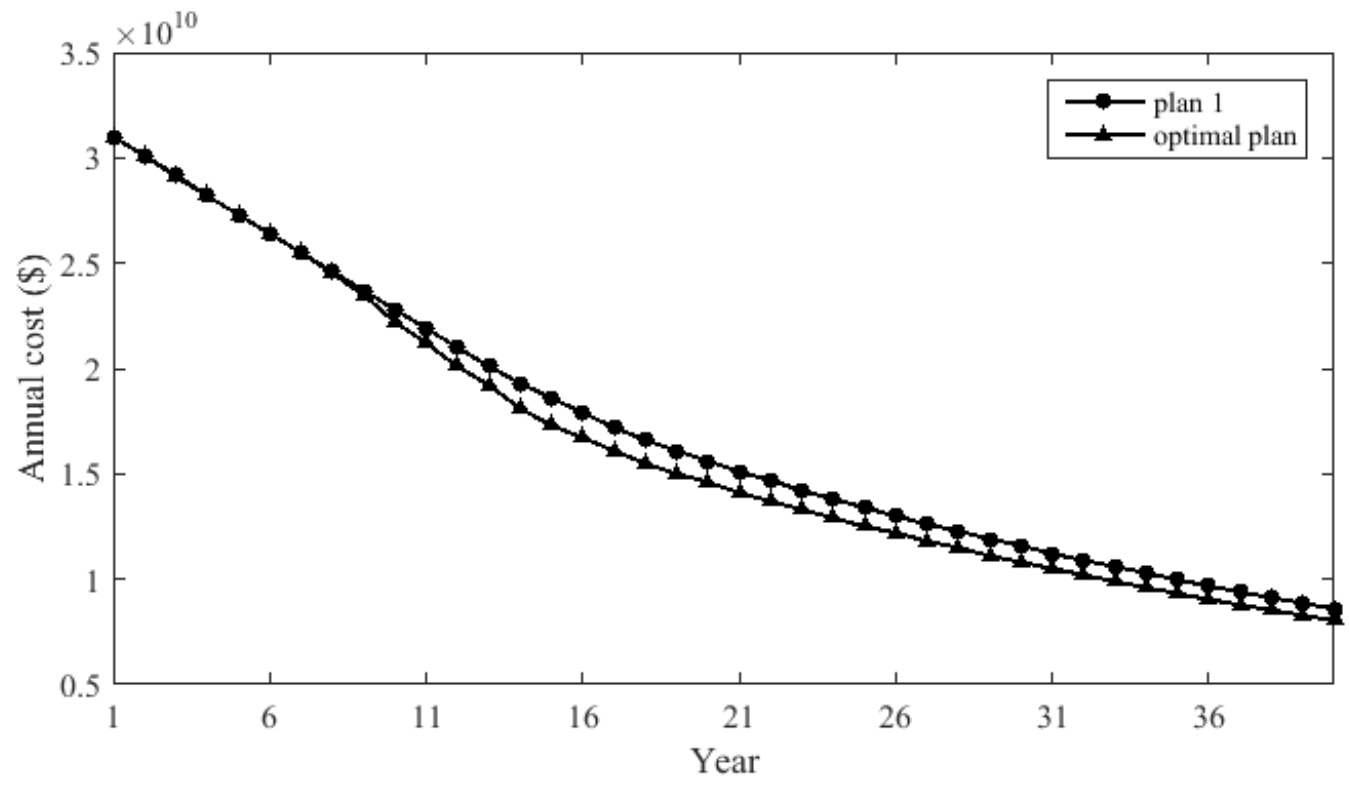

Figure 12 Evolution of annual cost under plan 1 and the optimal plan

\section{Concluding Remarks}

This paper proposes a mathematical procedure to optimally deploy AV lanes considering the endogenous AV market penetration. Given AV lanes deployed in a general road network, the 
flow distributions of both CVs and AVs are captured by a multi-class network equilibrium model. Further, a diffusion model integrating the net benefit derived from deploying AV lanes is applied to forecast the evolution of AV market penetration over time. Based on the network equilibrium model and the diffusion model, a time-dependent deployment model is further formulated to optimize the deployment plan of AV lanes. The deployment plan indicates when, where, and how many AV lanes to be located. The optimization model formulated is a mathematical problem with complementarity constraints, and an efficient active-set algorithm is applied to solve it. Numerical examples are presented to validate the proposed deployment model, and to demonstrate the importance of designing an appropriate deployment plan. Moreover, sensitivity analyses for various critical parameters are conducted. Results show that (1) AV lanes should be deployed following a progressive process instead of a radical one; (2) AV lanes should not be widely deployed until the AV market penetration reaches a relative high level (e.g., more than 20\%); (3) lower additional annual cost and VOT for AVs, higher unsafety factor for using CVs, and higher number of annual trips have positive impact on promoting the AV adoption, while the variance of the per-lane capacity of AV lanes has little impact.

A follow-up work can be the investigation of the parameters used in the proposed models, in order to more accurately forecast the AV market penetration. For example, the unsafety factor may be obtained via conducting surveys. In addition, since drivers are heterogeneous in terms of VOTs in reality, and those with higher VOTs are expected to adopt AVs earlier, another work can be carried out to expand the current model along this line.

\section{Acknowledgements}

The research is partially supported by grants from the U.S. National Science Foundation (CMMI-1362631; CMMI-1562420), the Southeastern Transportation Research, Innovation, Development and Education Center (STRIDE), and the Natural Science Foundation of China (71401025; 71501107). Also, we want to thank the three anonymous referees for their valuable comments. 


\section{References}

2025AD, 2016. https://www.2025ad.com/in-the-news/blog/japan-driverless-cars-in-2020/ (accessed May 24, 2016).

Gao, Z., Wu, J., and Sun, H., 2005. Solution algorithm for the bi-level discrete network design problem. Transportation Research Part B, 39, 479-495.

Chen, Z., He, F., and Yin, Y., 2016. Optimal deployment of charging lanes in transportation networks. Transportation Research Part B, 91, 344-365.

Google Self-Driving Car Project, 2016. https://www.google.com/selfdrivingcar/ (accessed May $24,2016)$.

Greenblatt, J. B., and Saxena, S., 2015. Autonomous taxis could greatly reduce greenhouse-gas emissions of US light-duty vehicles. Nature Climate Change.

He, F., Wu, D., Yin, Y., and Guan, Y., 2013. Optimal deployment of public charging stations for plug-in hybrid electric vehicles. Transportation Research Part B, 47, 87-101.

He, F., Yin, Y., and Zhou, J., 2015. Deploying public charging stations for electric vehicles on urban road networks. Transportation Research Part C, 60, 227-240.

Huang, H. J., and Li, Z. C., 2007. A multiclass, multicriteria logit-based traffic equilibrium assignment model under ATIS. European Journal of Operational Research, 176(3), 1464-1477.

Lavasani, M., Jin, X., and Du, Y., 2016. Market Penetration Model for Autonomous Vehicles Based on Previous Technology Adoption Experiences. In Transportation Research Board 95th Annual Meeting (No. 16-2284).

LeBlanc, L.J., 1975. An algorithm for the discrete network design problem. Transportation Science, 9, 183-199.

Levin, M. W., and Boyles, S. D., 2016a. A cell transmission model for dynamic lane reversal with autonomous vehicles. Transportation Research Part C, 68, 126-143.

Levin, M. W., and Boyles, S. D., 2016b. A multiclass cell transmission model for shared human and autonomous vehicle roads. Transportation Research Part C, 62, 103-116.

Luo, Z. Q., Pang, J. S., and Ralph, D., 1996. Mathematical Programs with Equilibrium Constraints. Cambridge University Press, New York, New York. 
Massiani, J., and Gohs, A., 2015. The choice of Bass model coefficients to forecast diffusion for innovative products: An empirical investigation for new automotive technologies. Research in Transportation Economics, 50, 17-28.

Mersky, A. C., and Samaras, C., 2016. Fuel economy testing of autonomous vehicles.

Transportation Research Part C, 65, 31-48.

National Highway Traffic Safety Administration (NHTSA), 2016.

http://isearch.nhtsa.gov/files/Google\%20-\%20compiled\%20response $\% 20$ to\%2012\%20Nov\%20\%2015\%20interp\%20request \%20-\%204\%20Feb\%2016\%20final.htm (accessed May 24, 2016).

Park, S. Y., Kim, J. W., and Lee, D. H., 2011. Development of a market penetration forecasting model for Hydrogen Fuel Cell Vehicles considering infrastructure and cost reduction effects. Journal of Energy Policy, 39, 3307-3315.

Shladover, S., Su, D., and Lu, X. Y., 2012. Impacts of cooperative adaptive cruise control on freeway traffic flow. Transportation Research Record, 2324, 63-70.

Tientrakool, P., Ho, Y. C., and Maxemchuk, N. F., 2011. Highway capacity benefits from using vehicle-to-vehicle communication and sensors for collision avoidance. In Vehicular Technology Conference (VTC Fall), 2011 IEEE, 1-5.

Wang, S., Meng, Q., and Yang, H., 2013. Global optimization methods for the discrete network design problem. Transportation Research Part B, 50, 42-60.

Wardrop, J. G., 1952. Some theoretical aspects of road traffic research. ICE Proceedings: Enginnering Divisions, 1, 325-362.

Wu, D., Yin, Y., and Lawphongpanich, S., 2011. Pareto-improving congestion pricing on multimodal transportation networks. European Journal of Operational Research, 210, 660-669.

Wu, D., Yin, Y., Lawphongpanich, S., and Yang, H., 2012. Design of more equitable congestion pricing and tradable credit schemes for multimodal transportation networks. Transportation Research Part B, 46, 1273-1287.

Wu, J. H., Florian, M., and He, S., 2006. An algorithm for multi-class network equilibrium problem in PCE of trucks: application to the SCAG travel demand model. Transportmetrica, 2, $1-9$. 
Yang, H., and Meng, Q., 2001. Modeling user adoption of advanced traveler information systems: dynamic evolution and stationary equilibrium. Transportation Research Part A, 35, 895-912.

Yin, Y., Lawphongpanich, S., and Lou, Y., 2008. Estimating investment requirement for maintaining and improving highway systems. Transportation Research Part C, 16, 199-211.

Zhang, L., Lawphongpanich, S., and Yin, Y., 2009. An active-set algorithm for discrete network design problems. Transportation and Traffic Theory 2009: Golden Jubilee 2009, 283-300.

Zhang, L., Yang, H., Wu, D., and Wang, D., 2014. Solving a discrete multimodal transportation network design problem. Transportation Research Part C, 49, 73-86. 